\title{
Optic nerve sheath meningioma
}

\author{
Mirela Dumitrita Ilie', Delia Adriana Parvu², Ionela Aurelia Ionescu', \\ Gabriela Mihailescu ${ }^{1,3}$ \\ ${ }^{1}$ Neurology Department, Colentina Clinical Hospital, Bucharest, Romania \\ ${ }^{2}$ Affidea - Hiperdia SA, Bucharest, Romania \\ 3"Carol Davila" University of Medicine and Pharmacy, Bucharest, Romania
}

\begin{abstract}
Optic nerve sheath meningiomas (ONSM) are rare, frequently unilateral, benign, slowly growing tumors, arising from the meningo-epithelial cells surrounding the optic nerve intraorbital or intercanalicular. Their localization directly impair vision and indirectly impair aesthetics, making a safe classical surgical intervention very difficult and recommending stereotactic fractionated radiotherapy. Women are more affected, the survival rate is good and imaging can sustain differential diagnosis with optic neuroglioma or orbital schwannoma, lymphoma or pseudo tumor.
\end{abstract}

Keywords: optic nerve meningioma, imaging, visual impairment, orbital tumor

\section{INTRODUCTION}

Optic nerve sheath meningiomas (ONSMs) are tumors surrounding the optic nerve and they are often associated with neurofibromatosis type 2 . They represent $2 \%$ of all orbital tumors and one third of all optic nerve neoplasms, most frequently unilateral and only $5 \%$ occurring bilateral $(1,2)$.

The triad : vision loss, optocilliary shunt vessels and optic atrophy seen at fundoscopy characterizes this type of tumor. Other common symptoms are: proptosis, headache, visual field impairment and diplopia. ONSMs are benign tumors with a slow growth and insidious symptom onset $(3,4,5)$.

They have a higher prevalence in women and the mean age for diagnosis is 40 years (1).

\section{CASE REPORT}

We present the case of a 54-year-old woman known with paroxysmal atrial fibrillation, high blood pressure and glaucoma who was seen for the first time 12 years ago by an endocrinologist, as she noticed an aesthetic change, due to a mild right proptosis, being afraid of unilateral exophthalmia. After performing a brain and orbital CT scan, she was diagnosed with a right optic nerve tumor. Imaging diagnosis was completed with a MRI examination and she was monitored yearly.

She complained of: diplopia, right proptosis and conjunctival edema, right periorbital chemosis, decreased visual acuity with blurred vision and impaired visual field in the right eye (right superior temporal quadrantanopia). The symptoms had a slow progression over the 12 years.

The neurological examination revealed decreased abduction, supraduction and infraduction of the right eye and diplopia.

The brain and orbital MRI showed a spindle-shaped, well delineated intraorbital mass measuring 2/1,7/1,6 cm, T1 and T2 - isointense compared to the optic nerve (Fig. 1, 2), with homogeneous and significant contrast enhancement in the $\mathrm{T} 1$ weighted images, gadolinium enhanced sequences, with no enhancement of the optic nerve ("tram-track" sign on axial images and "doughnut" sign on coronal images), surrounding the retro bul- 


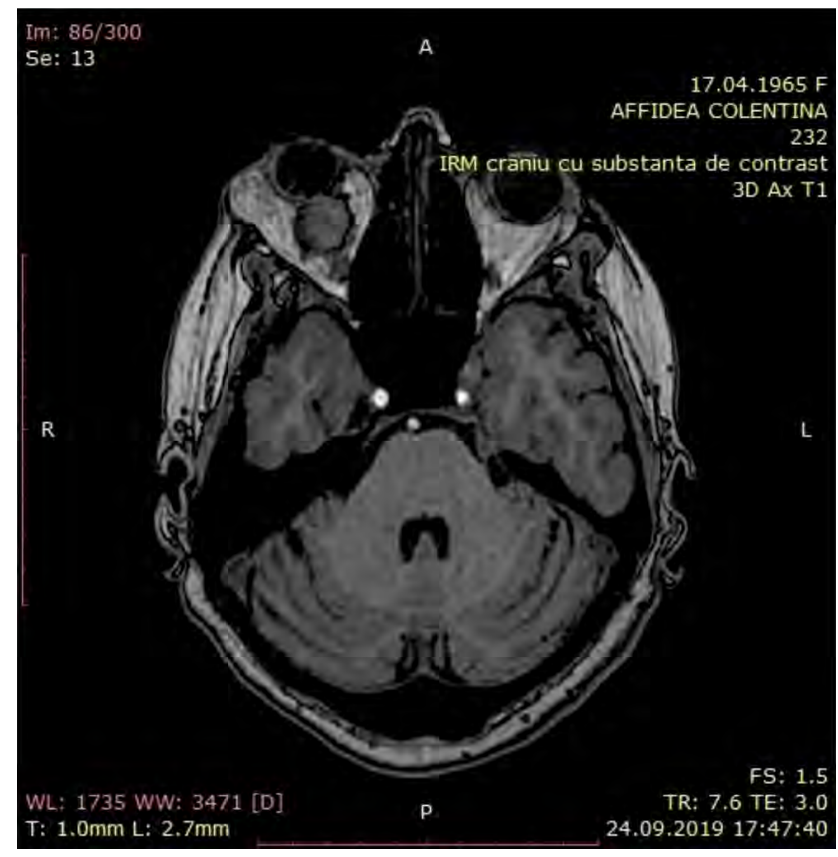

FIGURE 1. Axial T1 - weighed image presents an isointense right orbital mass

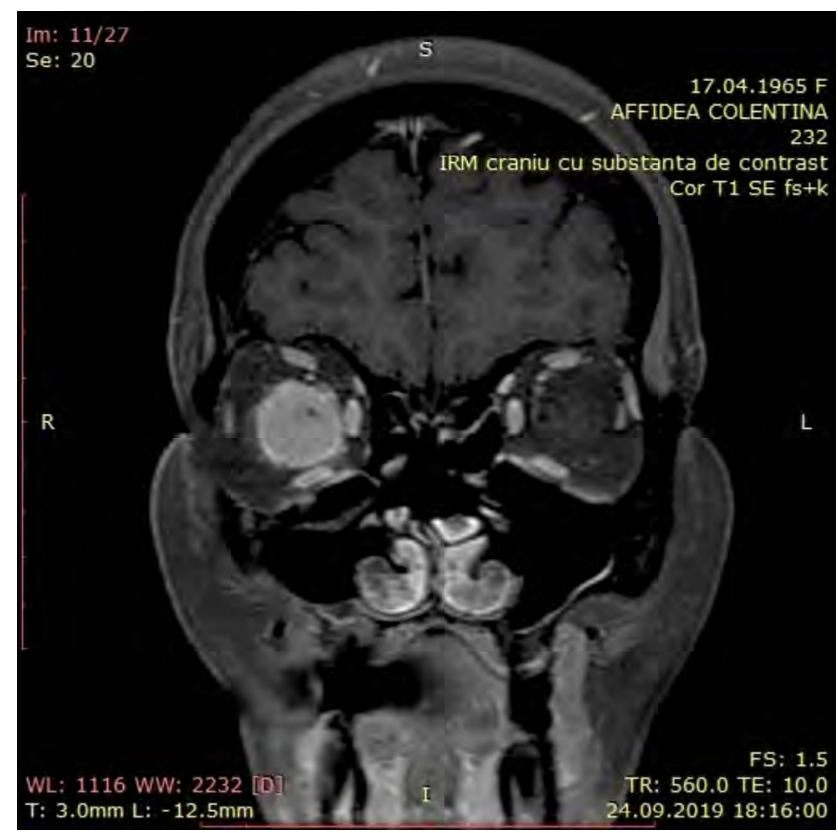

FIGURE 3. Coronal T1 - weighed image shows the lack of enhancement of the right optic nerve ("doughnut" sign)

bar segment of the right optic nerve (Fig. 3, 4). There is no significant difference between the images on the MRI examination performed in 2007 and in 2019.

Taking into account the clinical evolution with slow progressive symptoms and the imaging MRI evolution, the tumor is considered an optic nerve sheath meningioma (ONSM).

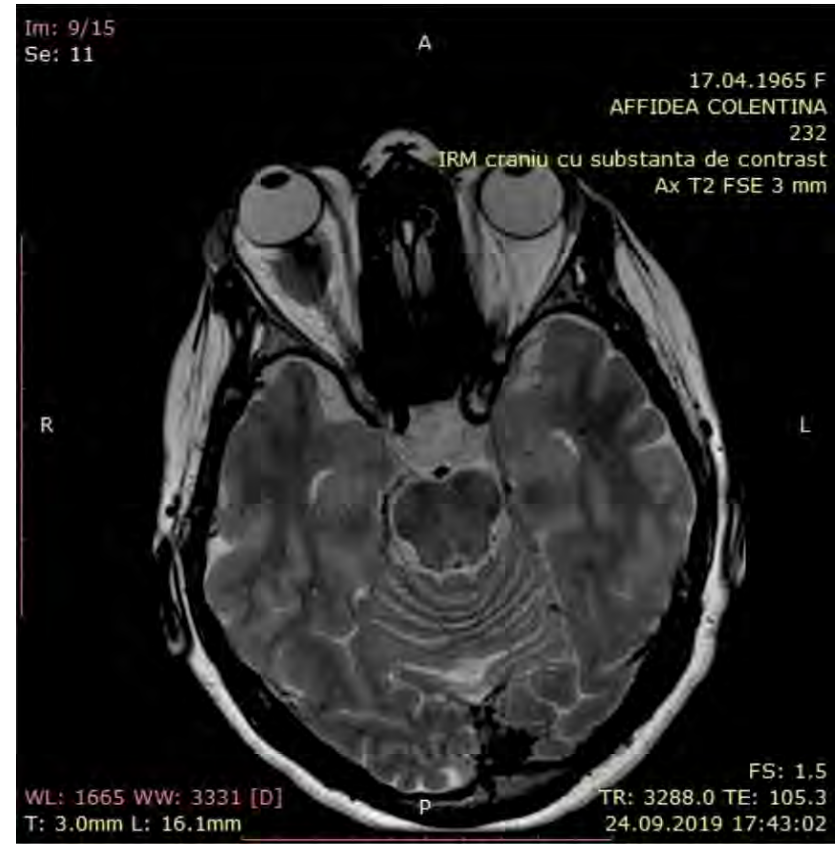

FIGURE 2. Axial T2 - weighed image presents an isointense homogeneous right orbital mass surrounding the optic nerve

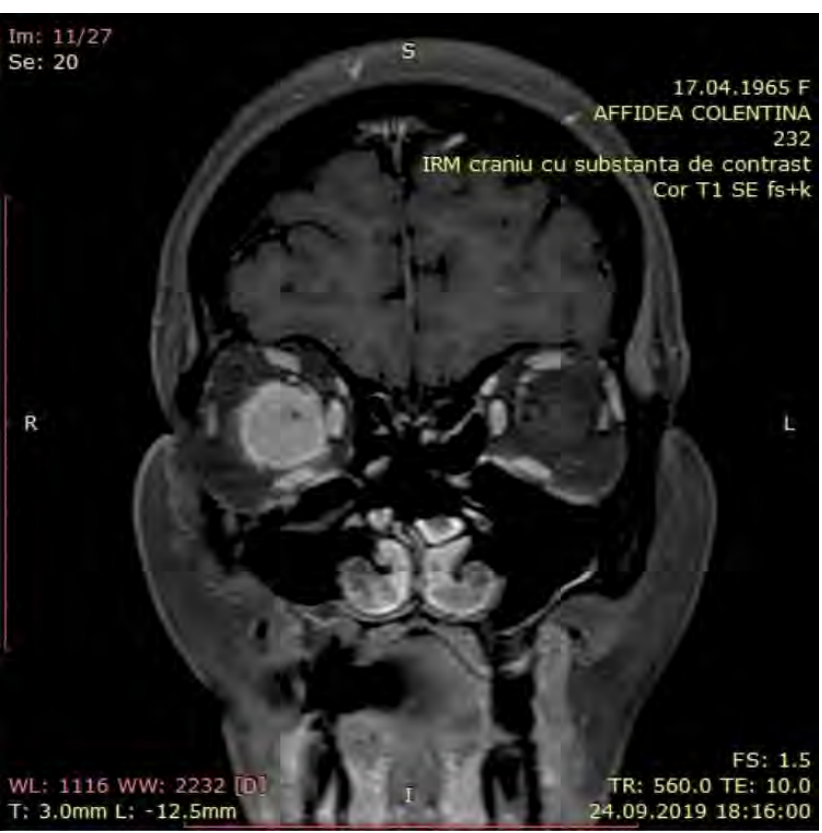

FIGURE 4. Axial T1 - weighed image shows the lack of enhancement of the right optic nerve ("tram-track" sign)

\section{DISCUSSION}

A differential imaging diagnosis should be made with: optic nerve glioma (enhancement of the optic nerve, more frequent in children), orbital schwannoma (heterogeneous enhancement with cystic components), orbital pseudo tumor (rapid-onset with painful proptosis and diplopia, enlargement of 
the extra ocular muscles on MRI), metastases (bone destruction, heterogeneous enhancement, rapid progression), orbital lymphoma (located especially in the upper temporal quadrant, moderate enhancement, rapid growth), optic neuritis (orbital pain, diffuse swelling of the optic nerve, T2- hyperintense, absence of a tumor) $(3,4)$.

If the visual function is normal, the ONSM will be just monitored. The patient should perform yearly a MRI examination. If the visual function de-

\section{REFERENCES}

1. Parker RT, Ovens CA, Fraser CL, Samarawickrama C. Optic nerve sheath meningiomas: prevalence, impact and management strategies. Eye Brain. 2018; 10: 85-99.

2. Patel BC, Najem K, Margolin E. Optic Nerve Sheath Meningioma. StatPearls Publishing, June 2019.

3. www.emedicine madescape.com - optic nerve sheath meningioma. creases, stereotactic fractionated radiotherapy is the treatment of choice. Surgical resection has an increased risk of visual loss and it is recommended only if the patient has disfiguring proptosis, severely decreased visual function or intracranial extension of the tumor $(2,6)$.

The survival rate at 5 years is $90 \%$ for typical meningiomas and $85 \%$ for atypical meningiomas (2).

Conflict of interest: none declared Financial support: none declared

4. www.radiopaedia.org - optic nerve sheath meningioma.

5. Jin J, Joo JD, Han JH et al. Optic Nerve Sheath Meningioma: Preliminary Analysis of the Role of Radiation Therapy. Brain Tumor Res Treat. 2018;6(1):8-12.

6. www.uptodate.com - optic nerve sheath meningioma. 